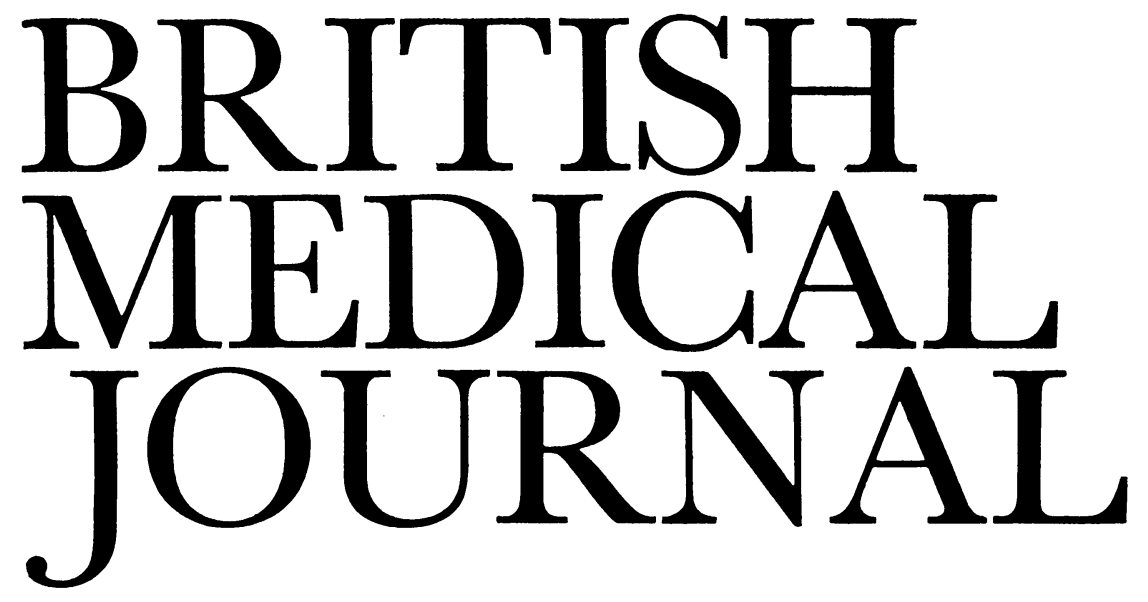

\title{
Hopes and realities in health care
}

The National Health Service presents a paradox. It is a Service which is getting better year by year, to judge by the statistical evidence, yet those working in it seem to believe that it is deteriorating. On the one hand, the statistics of spending and activity suggest that the NHS has increased the scale and scope of its services over the past decade. On the other hand, the perceptions of the providers-doctors, nurses, and otherslargely contradict the statistics and suggest that standards and quality have been slipping. While the government preens itself on the evidence of improvement in the NHS, the professions denounce the inadequacy of existing funding. Who is right?

This question is prompted by the publication of Health Care and its Costs by the Department of Health and Social Security. In effect, this is the Department's response to its professional and political critics - an endeavour to demonstrate that far from deteriorating, the performance of the NHS has improved despite the squeeze on spending and despite the extra demands generated by an aging population. It brings together information about both costs and activities from a variety of published sources to provide a picture of progress over the past decade.

In making out the case that the NHS should be seen as a success story the DHSS has assembled some impressive statistical evidence. In the decade from 1971-2 to 1981-2 the input of real resources into the NHS went up by $60 \%$. Even in the half decade from 1976 onward, when the combination of national economic crisis and cash limits was beginning to bite, the Health Service budget continued to expand, even if at a slower rate. The number of doctors increased at an annual average of $2.7 \%$; that of nurses rose by $1.5 \%$ (after making allowance for the fall in the number of hours worked). Moreover, the "output" of the NHS-the number of patients treated-increased even faster. While the input of resources rose by an average of $1.6 \%$ a year from 1976 to 1981 , the output of the service increased by $2 \cdot 2 \%$. This reflects, argues the DHSS, improved efficiency and productivity. After rising during the first half of the 1970s costs per acute case fell during the second half and the trend seems to have been maintained in the 1980s. The rise in costs implied in treating more elderly patients and using more sophisticated techniques of diagnosis and treatment was offset by a fall in lengths of stay and a slight reduction in hotel costs.

Over the same period, the DHSS claims, "standards of care were improved in the long stay sector," While the costs per acute case fell, the cost of treating patients with mental illness and handicap rose in line with the DHSS strategy, set out in a succession of documents on priorities, of pumping more resources into these sectors. Staffing ratios improved, as did the provision of day and community services. In retrospect, the much criticised 1974 reorganisation of the NHS appears to be at least partly vindicated: not only does the planning machinery then set up seem to have nudged NHS services in the direction laid down by the DHSS, if rather more slowly than intended, but the new management structure appears to have promoted efficiency. Perhaps Sir Keith Joseph recanted too soon: Health Care and its Costs provides a warning against rushing into quick judgments in a complex service like the NHS where the impact of any changes-whether financial or organisational -is likely to be slow, diffuse, and difficult to disentangle from other developments.

Even given the time lag between policy changes and their translation into statistical measures of service provision, however, there still remains a puzzle. If the NHS is indeed the success story presented in the DHSS's prospectus, why is there such widespread disquiet among those actually providing the Service? To return to the question with which we started, what explains the apparent contradiction between official statistics and professional perceptions? One answer would seem to be that this contradiction reflects the tension between two quite different ways of looking at the NHS and attempting toassess its performance. Such an exercise which uses different criteria of evaluation is likely to yield different conclusions. ${ }^{2}$

For doctors and other professionals in the NHS the starting point for making any judgment about the adequacy or otherwise of the services provided is to ask about the quality of care received by individual patients-the extent to which the potentials of modern diagnostic and therapeutic techniques can be mobilised on behalf of the individual. This dimension, however, is not caught by the official statistics. As Mr Norman Fowler concedes in his foreword to Health Care and its Costs, the figures cannot measure "the human care for each individual patient and the concern for quality that must be central to any health service worthy of the name."

This concern is, inevitably, not reflected in statistical evidence about the quantity of services provided or the numbers of staff employed. Yet it would be wrong to dismiss the DHSS's case on this account. The quantity of services 
provided may be only one criterion for assessing the performance of the NHS, yet it is clearly an essential one: we would not think much of a health care system which improved the quality of care provided to a diminishing proportion of the relevant population. Indeed, it might be argued that, in a period of national economic stringency, it may be more important to maintain access to health care than to improve its quality. Perhaps the main criticism of Health Care and its Costs is not that it puts too much reliance on quantitative information, but that it does not provide an advanced enough analysis of the figures. In particular, crude totals of service activity do not provide a picture of how access to services for specific population groups, with specific needs, has changed over time, or of the variations in the availability of services in different parts of the country. In this respect the document compares poorly with the DHSS's own, earlier studies of the different sectors of the NHS, which were designed to analyse problems rather than to present a success story. ${ }^{3-5}$

There are also some technical and conceptual problems about interpreting the data in Health Care and its Costs. Consider, for example, the improvement in productivity-as measured by the fall in the cost per acute case-of which the document makes so much. As a technical appendix points out, the figures represent average case costs for "all cases in hospitals classified as acute, mainly acute or partly acute." It is thus an extremely heterogeneous category and includes psychiatric, geriatric, and maternity care provided in such hospitals. This would seem to indicate the need for caution in drawing any conclusions without further, more detailed analysis. Moreover, it is always possible to increase productivity - at least by shortening lengths of stay-by transferring the burden of care from the NHS to the family.

This point is both obvious and familiar, but it needs underlining since the government's spending plans ${ }^{6}$ are based on the assumption that it is feasible to squeeze annual "efficiency savings" of $0.5 \%$ out of the NHS's budget, year after year, and there is a danger that the figures in this document may be misused to argue that painless savings are possible simply by increasing productivity. Equally it is clear that the main responsibility for cutting costs and improving productivity will rest on the medical profession. Health Care and its Costs does not explicitly make this point: instead, it emphasises, quite rightly, the need to reduce administrative and hotel costs. Nevertheless, the figures presented in the document speak for themselves. Most of the money in the NHS is spent on those items-such as medical and nursing salaries, drugs, and dressings-where medical decisions directly determine how efficiently or productively resources are used. In contrast, while conceivably there may be greater scope for improving efficiency in the delivery of hotel services or administration, even large proportional savings will produce only relatively modest sums compared with the expenditure on the patient care services. Of course, the figures of increasing productivity would suggest, to the extent that these can be taken at face value, that the medical profession has already responded to the pressure to economise. Indeed one possible, if partial, explanation of the widespread perception of inadequacy within the NHS may lie precisely in the fact that it is the professional providers who have to carry the day to day strain of seeking to match rapidly expanding demands to the slow trickle of increasing resources. As Health Care and its Costs concedes, by itself the process of "medical innovation" requires an annual rise of $0.5 \%$ in the NHS budget-although it would be interesting to see the precise calculations and assumptions on which this, perhaps conservative, estimate is based.
The way in which the figures are presented in the document $\underline{\square}$ may gloss over some of the problems caused by increasing demands, particularly demographic ones. Health Care and its Costs rightly emphasises the extent to which the NHS is now dominated by the problems of the over $65 \mathrm{~s}$. But by emphasising $\widehat{\widehat{\Omega}}$ activities - the number of cases treated, the number of beds $\overline{\bar{J}}$ provided, and so on-the evidence, by definition, tells us nothing about non-activity: what is not done. For example, possibly the growth of the private sector reflects the in- 2 creasing concentration of the NHS on the elderly and the consequent deterioration of services for the younger, working $\stackrel{\vec{S}}{\rightarrow}$ population. More information on this point is surely desirable in any assessment of the NHS's overall performance.

There are other reasons, too, why Health Service profes- $\frac{D}{\bar{D}}$

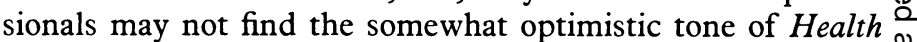
Care and its Costs convincing. Some of these reasons spring क from the very nature of the NHS; some reflect the special $\vec{\circ}$ circumstances of the 1980s. Tension between politicians and $\overrightarrow{\vec{\omega}}$ professionals is built into the very structure of the NHS. Politicians delegate responsibility for rationing scarce resources $\frac{5}{3}$ to professionals. They are, in effect, diffusing blame for inadequacy by turning national political decisions about taxing $\stackrel{\circ}{\circ}$ and spending into individual clinical decisions about whom to treat how. In turn, therefore, professionals have what Enoch Powell once described as a "vested interest in denigration." $7 \vec{\overrightarrow{ }}$ Reflecting on his experience as minister of health, 20 years ago, he wrote: "Anyone in the National Health Service below the minister, from the chairman of a hospital board to a nursing $N$ auxiliary, who professed himself satisfied with what was being $\rightarrow$ spent could not unreasonably be represented as a traitor to his colleagues, his profession and his patient-on the basis, $\vec{\varphi}$ namely, that more money means improvement. And that complaint and dissatisfaction are essential to extracting more money."

The special circumstances of the 1980s have, if anything, given extra point to Powell's observation. For they have seen a collision between inherited policies, forged in the days when it $\stackrel{\mathbb{Q}}{2}$ made sense to be optimistic about the prospects of both the $\overrightarrow{\overrightarrow{0}}$ British economy and the NHS budget, and the new reality of a 3 stagnant economy. The policies remain, as Health Care and its Costs confirms, to give priority to deprived geographical areas and patient groups. But the new circumstances of financial stringency mean that strategies designed to be implemented painlessly, by allowing the deprived regions and deprived $\frac{5}{3}$ services to grow faster than the rest, can often be carried out only by cutting the relatively "affluent." In particular, the $\frac{O}{3}$ attempt to secure a fairer geographical distribution of resources, 0 by applying the RAWP approach not only to the regions but also within them, means that services actually have to be cut in $\mathscr{N}$ those districts which are deemed to be overprovided according $\frac{D}{0}$ to the formula used. ${ }^{8} \mathrm{~A}$ justified sense of local crisis is therefore perfectly consistent with statistical evidence which suggests 0 that, nationally, the NHS is offering better services to more people.

Such reservations aside, however, the publication of Health $\stackrel{0}{\frac{0}{\varnothing}}$ Care and its Costs can be welcomed without reservation in one $\stackrel{\oplus}{\rightarrow}$ important respect. The very fact that $\mathrm{Mr}$ Fowler has felt it 0 both necessary and desirable to defend the performance of the NHS, and to insist that it is doing well, gives credence to his $\stackrel{\mathbb{P}}{\stackrel{P}{P}}$ statement in the preface that "the government's policy is to $\varrho$ maintain its commitment to the National Health Service so that the patient can receive the best possible care." If the government were not so committed and if it did not feel? vulnerable to political charges of neglecting the NHS it wouldō․

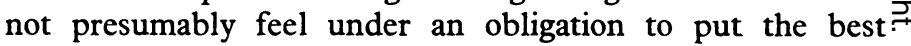


possible gloss on the performance of the NHS. Given such a commitment, the aim now should be to persuade $\mathrm{Mr}$ Fowler to live up to his aim and to ensure that the patient does indeed receive the best possible care.

Department of Health and Social Security. Health care and its costs. The development of the National Health Service in England. London: HMSO, 1983.

2 Klein R. Performance evaluation and the NHS. Public Administration 1982; 60:385-407.

${ }^{3}$ Department of Health and Social Security. Report of a study of the acute hospital sector. London: DHSS, 1981.

${ }^{4}$ Department of Health and Social Security. Report of a study on community care. London: DHSS, 1981.

5 Department of Health and Social Security. Report of a study on the respective roles of the general, acute, and geriatric sectors. London: DHSS, 1981.

${ }^{6}$ Chancellor of the Exchequer. The government's expenditure plans 1983-4 to 1985-6. Cmnd 8789. London: HMSO, 1983.

7 Powell, E. Medicine and politics. London: Pitman, 1966.

${ }^{8}$ Department of Health and Social Security. Sharing resources for health in England: report of the resource allocation working party. London: HMSO, 1976.

\section{Fat and cancer}

Fat can be carcinogenic in animals, and increasingly it is referred to as a cause of human cancer, particularly of the colon and breast. Indeed, the United States National Research Council judged that the evidence for this was stronger than for any other dietary constituent and sufficient to recommend that the consumption of both saturated and unsaturated fat should be reduced. ${ }^{1}$ It therefore seems timely to review the evidence, even if briefly, for fat as a cause of human cancer.

Mortality from both cancer of the colon and cancer of the breast in different countries shows a strongly positive correlation with the corresponding consumption of fat per head, ${ }^{2-6}$ which persists after controlling for several other national variables. ${ }^{6}$ Within countries the relationship has been upheld for breast cancer in five ethnic groups in Hawaii (though not for colonic cancer) $)^{7}$ and also in different parts of Japan. ${ }^{8}$ Moreover, the incidence of these cancers has increased in Japan ${ }^{8}$ and in Japanese migrants to the United States, ${ }^{910}$ and both populations have increased their fat consumption. Such data are inevitably crude and may reflect indirect relationships; perhaps their main value is to indicate the need for more sensitive studies of subjects not only for these cancers but also for cancers of the endometrium, prostate, and ovarywhich are also (though less strongly) correlated with fat in international comparisons. ${ }^{6}$

Studies of individuals within populations can, however, be more sensitive only if within those populations the differences in long term fat intake are large enough to cause substantial variation in the risk of disease. Even then dietary differences may not emerge because of inaccurate recall of past diet. Perhaps because of these factors studies of patients with colonic cancer and of controls ${ }^{31-16}$ have with few exceptions $^{1112}$ found no significant differences in fat intake. Although a United States case-control study ${ }^{17}$ found no difference in fat intake, two studies in Canada ${ }^{1819}$ found that compared with controls patients with breast cancer reported eating more fat; nevertheless, these require cautious interpretation, for in one ${ }^{18}$ bias may have been introduced by concentrating on diet around the time of diagnosis, a more memorable period for the patients, and in the other ${ }^{19}$ different methods were used for interviewing the patients and the controls.
A prospective study in Japan has reported a positive relationship between fat and cancer, ${ }^{8}$ but the only such study in a Western country, which was of nuns in strict religious orders in Britain, ${ }^{20}$ found no appreciable reduction of either colorectal or breast cancers among those with a low intake of fat. This negative result suggests that fat intake in adult life is not of major importance but gives, of course, no information about the relevance of fat intake in childhood and adolescence. Some groups of vegetarians tend to have a lower intake of fat than the average, but the only published studies of cancer among vegetarians concern Seventh Day Adventists in California, who apparently have a fat intake ${ }^{21}$ (and also a mortality from breast cancer ${ }^{22}$ ) close to the average in the United States. Despite their normal fat consumption, their incidence of colonic cancer is low, as it also is in United States Mormons, ${ }^{23}$ who do not restrict their meat intake, so other factors are clearly concerned. After a claim that trials of diets containing polyunsaturated fat supplements increased the total incidence of cancer, Ederer and his colleagues examined the findings in all five trials with relevant and available data but overall found no evidence of any influence on the risk of cancer. ${ }^{24}$

To turn to measures that are based on objective biochemical tests instead of questionnaires, the concentrations of faecal bile salts and neutral and acid steroids are indirectly related to fat intake. Hence, like average fat intake itself, the amounts in faecal samples from individuals in different countries show a positive relation with the corresponding mortality from cancer of the colon and breast. Because of some apparently associated differences in the intestinal flora this initially suggested a simple hypothesis - that colonic cancer was due to carcinogens formed by the interaction of nuclear dehydrogenating clostridia and fatty acid residues. ${ }^{25}$ This hypothesis has not been supported by the results of subsequent studies. A theory of the aetiology of cancer of the breast involving faecal oestrogens produced in a similar way was also proposed. ${ }^{26}$ Faecal steroids in patients with cancers of the colon ${ }^{27-32}$ and breast ${ }^{33} 34$ compared with those in controls have, however, shown inconsistent differences-though in cancer of the colon the results are likely to have been substantially affected by the disease. At least one prospective study is in progress but as yet no results have been published. The higher concentrations of faecal steroids observed in detailed studies in New York ${ }^{35}$ and Denmark ${ }^{36} 37$ (with a high incidence of cancer of the colon) as compared with Finland ${ }^{35-37}$ (which has a low incidence) are often quoted as supporting a role for fat. Nevertheless, the results of these studies are more relevant to the role not of fat but of fibre, for the total intake of fat (and also the total excretion of faecal steroids) was similar in these areas, and faecal steroid concentration was lower in Finland chiefly because the intake of (pentosan) fibre and consequently the faecal bulk was greater.

Blood lipid concentrations are also indirectly related to fat consumption and have been subject to detailed study particularly in relation to coronary artery disease. In 1974 Rose and his colleagues ${ }^{38}$ reported their unexpected observation of an inverse relation (in men) between serum cholesterol concentrations and subsequent mortality from cancer of the colon, and later other workers noted a similar relation for total mortality from cancer. In 1981 a working party of the United States National Institutes of Health considered this subject, ${ }^{39}$ mainly in relation to cancer mortality as a whole, finding that none of the 16 prospective studies examined showed a positive relation but that no fewer than 24 showed an inverse relation (all in men). More recently an international collaborative group has investigated the question ${ }^{40}$ with 